\title{
ENHANCING ENTERPRISE COLLABORATION BY USING MULTIFACETED SERVICES
}

\author{
Sodki Chaari ${ }^{1,2}$, Loubna Ali ${ }^{1}$, Frédérique Biennier ${ }^{1}$, Joël Favrel ${ }^{1}$, \\ Chokri Ben Amar ${ }^{2}$ \\ INSA de Lyon - LIESP, Lyon, FRANCE \\ \{sodki.chaari, loubna.ali,frederique.biennier, joel.favrel\}@insa-lyon.fr \\ ENI de Sfax-REGIM, Sfax, TUNISIA \\ Chokri.benamar@enis.rnu.tn
}

\begin{abstract}
In fast changing markets, dynamic collaboration ability involves establishing and "enacting" business relationships on the fly in an adaptive way. Such dynamic organization relies on interoperable and agile IT solutions. A contemporary approach for addressing these critical issues is the Service Oriented Architecture which can be introduced to implement opened and flexible information system. Accordingly, collaborative processes can be implemented as a chain of services resulting of a service composition process. Nevertheless, combining "directly" elementary IT services from different enterprises may lack of security (as the different enterprises can get information of the partner IS organization) and leads to highly complex system due to the IT service granularity. To overcome these limits, we have developed a service oriented enterprise model which transforms enterprise structure as a set of well defined Virtual Service. Virtual Services are used to orchestrate more elementary IT services and are combined together to form the service chain according to different policies. As contractual relationships between enterprises are concerned, a particular attention must be paid on the service level agreements to support an end to end convenient quality of service level. That is why, we propose to couple the Virtual Service description to a dynamic service level management system, orchestrated thanks to elementary agents.
\end{abstract}

\section{CONTEXT}

Nowadays, organizations are more and more centred on their core competencies and outsource secondary tasks. This outsourcing policy leads to stronger interorganisational relationships often based on service customer/service supplier relationships to adapt and increase continuously the global performance level and gain a competitive advantage. Consequently, enterprises focus has shifted from the improvement of their internal organizational level to the outsourcing strategy and the inter-organizational level. The combination of service consumer and service provider can be seen as global virtual enterprise which can be introduced to a third party as a single entity.

Such collaborative organizations can be more or less stable, depending on the parties contracting conditions and on the environment context which may require more or less flexibility and agility (Kutvonen et al., 2005). This agility constraint, especially in fast changing environments, involves continuous adaptation of both the enterprise internal organization and collaboration partners selection. This context leads to the emergence of dynamic virtual markets in which partners are selected for short or midterm collaboration (Bartelt and Lamersdorf, 2001). Consequently, a highly dynamic approach is required to create or retain a competitive position for

Chaari, S., Ali, L., Bicnnicr, F., Favrel, J., Amar, C.B., 2007, in IFIP International Federation for Information Processing, Volume 243, Establishing the Foundation of Collaborative Networks; eds. Camarinha-Matos, L., Afsarmanesh, H., Novais, P., Analide, C.; (Boston: Springer), pp. 521-528. 
such an organization where service consumers dynamically determine which service providers will be selected to support the collaborative business process enactment. We define such a collaborative strategy as dynamic business process collaboration. This paradigm implies dynamic selection, contracting, composition and execution of business services.

Several works have addressed this efficient and fast dynamic business process collaboration: from the structured electronic collaboration between organizations achieved by Electronic Data Interchange (EDI) to frameworks enabling composition, service orchestration, contract enactment, executing and monitoring business services. Nevertheless, as the EDI approach focus on structured data interchange trough electronic transport media (Wob, 2000), it leads to a static connection between partners and not so much on process integration in dynamic business service collaboration. On the other hand, several approaches have been proposed to support inter-organization collaboration, by extending traditional Workflow Management System (WfMS) technology to distributed, Internet-based scenarios. For example the WISE project (Lazcano et al., 2000) proposes an infrastructure to support inter-organizational process: collaboration processes (named virtual business processes) are built thanks to black box services published by potential partners in different registries. Nevertheless as the resulting process consists in the fixed orchestration of "concrete" IT services, it does not exhibit flexibility or agility and a particular attention must be paid on Information System (IS) interoperability.

To support this interoperability requirement, standards are emerging and allow a more dynamic connection between organizations, typically through the use of Internet and XML technology such as the frameworks based on ebXML (Choi et al., 2004) or RosettaNet (Sundaram and Shim, 2001). Nevertheless, these approaches lack of process integration which may lead to inconsistent service chains.

Focusing on process integration leads either on a multiple Workflow management strategy (as the cross flow project) or on a service composition approach (as in eFlow project). Multi Workflow management strategy, as proposed in the CrossFlow project (Grefen et al., 2000) involves the Workflow connection according to a service outsourcing logic. In this case, a service specification is detailed thanks to a contract described in a XML-based specification language. The contract provides the structure of the Workflow which implements the service in combination with other useful ones. The meta-model of both the inter-organizational process and the service structure is the WfMC process meta-model. A service matchmaker (based on the CORBA Trading Service) allows retrieving contract satisfying specific requirements. Services are managed by Workflow management systems and the inter-organization process enactment is performed by Cooperative Support Services (CSS's), which link the different Workflow management systems of the involved organizations through appropriate Proxy Gateways.

On the other hand, the service composition approach as in the eFlow (Casati and Shan, 2001) involves specifying, enacting and monitoring composite e-Services. A composite service is described as a process schema (i.e. a graph which combines basic or composite services and defines the order of execution among the nodes in the process). Service heterogeneity is taken into account as eFlow provides adapters for services that support various B2B interaction protocols such as RosettaNet.

Nevertheless, such collaborative strategy relies mostly on the Information System agility and interoperability. These constraints can be partially taken into 
account thanks to Service-Oriented Architecture (SOA) and particularly the Web service. Such SOA based IS can reinforce a business environment with a flexible infrastructure and processing environment. thanks to independent, reusable automated business process and systems functions (services) to either end-user applications or to other services distributed in a network, via published and discoverable interfaces (Alamri et al., 2006, Alonso et al., 2004, Papazoglou, 2003). Nevertheless, this approach fits well the IT implementation constraint but needs improvements to fit the collaborative enterprise requirements.

Accordingly, we extend this approach to the global enterprise level, and not only to the IT level. The result is a flexible, agile, managed SOA ecosystem that supports dynamic enterprise collaboration. This architecture is based on SOA and extends it to a Service Oriented Enterprise (SOE) (Chaari et al., 2006). Typically, a Service Oriented Enterprise is an enterprise which implements and exposes its business processes through a set of well defined business services. Such services are organized into a multifaceted service called Virtual Services (VS) which bridge the businessoriented world of dynamic business collaboration and the Web service technologies. The main issue of this work consists in improving the IT service structure and functionalities to enable dynamic and flexible business process collaboration through VS composition (section 2) including end to end service level management (section 3).

\section{VIRTUAL SERVICE ARCHITECTURE}

The central feature of the Service Oriented Enterprise is the alignment of both IT and business sides. In fact, we define two abstraction levels: IT services (i.e. technical services) and business services. On the IT side, Service Oriented Architecture can be built thanks to traditional methods so that a well defined set of IT services can be implemented and orchestrated. Business services are associated to the enterprise business functionalities. They correspond to a set of fine-grained services called business objects and more coarse-grained services called business components. These business components encapsulate the Workflow logic of an activity or the business process they expose. Typically business components are made of business objects which are composed of lower level services, i.e. IT services.

This integration logic aims both at reducing the global complexity (as the Virtual Service orchestrates several concrete services) and increasing the IS security (as the VS encapsulates the lower level IS components and the VS publication is controlled by well defined security policy). As a result, the SOE offers a set of Virtual Services which represent a combination of related IT/Business services to a single logical service. A virtual Service provides no direct business function but offers a global view over those services for customers. Consequently, a Virtual Service can be processed and combined as a traditional Web service, but as its objective is not to define new application programming interfaces (APIs) or new standards; it enhances the Web service concept. The VS builds a higher-level structure which hides the traditional discovery, selection and composition functions so that it simplifies the service deployment by reducing the orchestration complexity. It also provides selfmanagement capabilities related to the service level agreement required.

Consequently, building a service chain relies on the Virtual Service composition process including a multi criteria partner and Virtual Service selection (for example 
these criteria can be: due date, costs management rules, partner trust, and required service level...) and the concrete service interconnection process. To simplify the VS selection, criteria are gathered in consistent policies described as XML documents. The global orchestration is first achieved by connecting the Virtual Service and then connecting the different IT service chains orchestrated by the different Virtual Services each of them orchestrating the related concrete services. The aggregation of different services belonging to a Virtual Service is achieved through Web service composition languages like BPEL (Andrews et al., 2003). The default model underlying BPEL is that an abstract process is shared among the partners but executable business process remains private to each partner. However, this does not suit the dynamic business process collaboration where service provider wants to provide explicitly information about internal process structure and evolution to partners and enable them to monitor and control an internal process. Developing adapted service monitoring functions involves connecting the service chain to the Service Level Agreements (SLA) chain. Nevertheless, actual SLA frameworks are often limited to elementary technical views and do not fit the service chain constraint. Consequently, we propose to extend the Virtual Service description by adding a Service Level Management (SLM) facet (figure 1), used to orchestrate management agents so that quality of service can be introduced as a key element in the service composition policy.

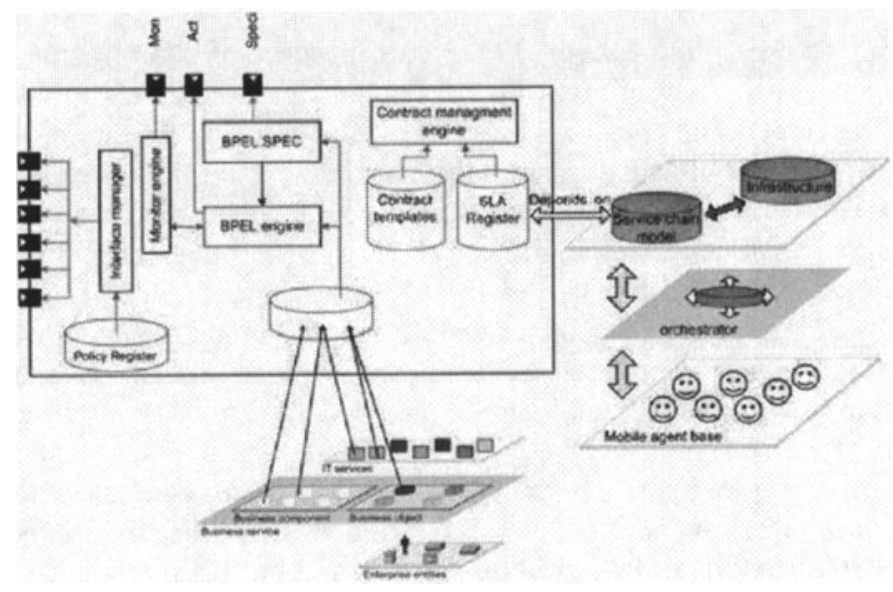

Figure 1: Virtual Service architecture

\section{A DISTRIBUTED AND DYNAMIC SLM}

While building a service chain, one must introduce a service management facet to control and monitor the global chain execution. Such end to end Service Level Management is a complex task which must be adapted dynamically to the current service chain, infrastructure capability.

SLM is the set of activities required to measure and manage the quality of service provided to end users (Bissel et al., 2000). SLM represents a broader framework for developing and executing Service Level Agreements (Engel, 1999, Verma, 1999). These works are mostly focusing on telecommunication services. 
While building the SLA chain describing the QoS requirements, a consistent quality monitoring system must be defined accordingly so that the end to end service level can be monitored.

To set the SLA recommendation of quality of service, Key Quality and Key Performance Indicators (KQI/KPI) are used to measure specific aspects of application or services performance. The global quality of service is described thanks to KQI aggregated in KPI. Then SLA is defined between interconnected services to define KPI goals (TheOpenGROUP, 2004). Consequently, contractual relationships between business application leads to a SLA chain (figure 2).

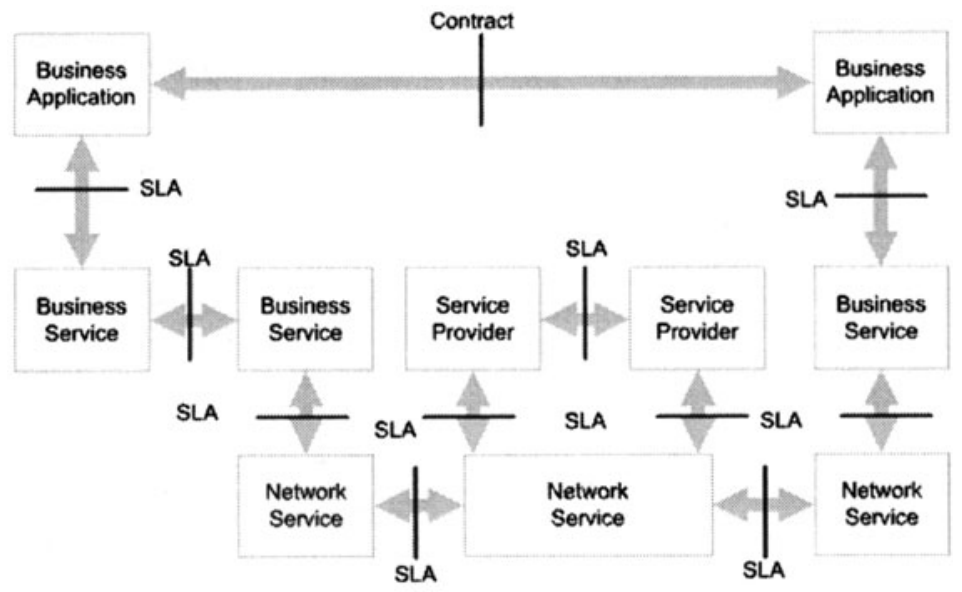

Figure 2: Expanded view of SLA in achieving the End-to-End SLA (TheOpenGROUP, 2004, page 21)

As the global service chain performance level is related to both the organization and the support infrastructure, we propose to include both organizational and operational (i.e. infrastructure related) information in the Service Level Management. Our system consists in three main interconnected parts (Ali et al., 2006): first, the process description area (organizational level), second, the monitoring information area (management level) and lastly the management process or the implementation area (infrastructure level). This architecture leads to a global service chain model (figure 3) used to guide the management service orchestration. As the organizational area is defined by the Virtual Service orchestration, we reduce this description to the distributed business process according to a Workflow organization, coupled to the information system description. Then, the Virtual Services implementation requires IT resources, i.e. concrete services. These resources (presented in the monitoring area), can be associated either to software (mostly application and information parts) or to hardware resources (workstations, servers, communication network equipments...). Consequently, different monitoring information (log files, equipment activity measures...) and convenient SLA patterns can be related to the exact “concrete service chain" (figure 3). 


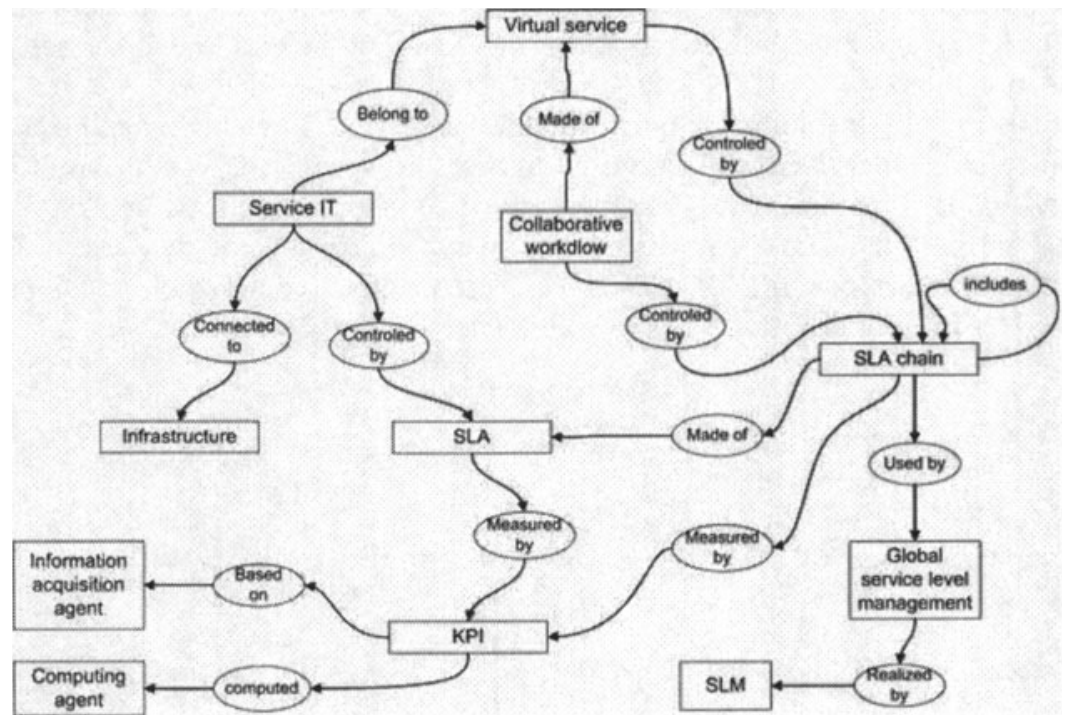

Figure 3: Service chain model

To fit the partner autonomy requirement as well as the end-to-end dynamic service chain requirement, a decentralized management organization must be set. Moreover, due to the agility constraints, an evolving management infrastructure must be implemented. To fit this requirement, we propose a mobile agent architecture including different agent types that can be orchestrated to set adapted management functions dynamically (figure 4).

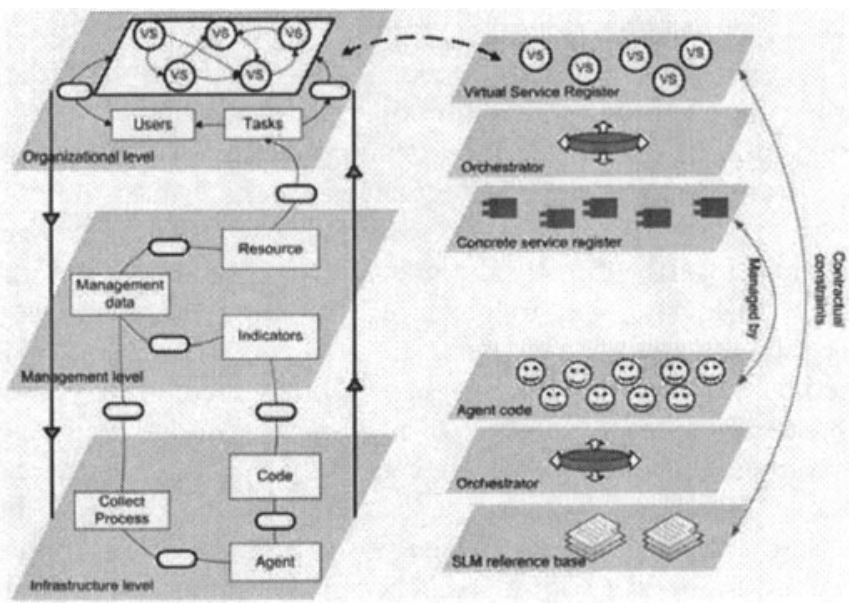

Figure 4: Multi-level monitoring system

Each management unit has to orchestrate the convenient service management agents according to the current context. Management features (both those dealing with the business activity management and those related to the infrastructure monitoring) are based on data collection, indicators building processes and actions 
(configuration, policy parameter adjustment...). Orchestrating the global management system consists in the convenient combination of three main types of agent instantiated from the agent patterns stored in the SLM reference data base:

- Itinerary agents are in charge of the "mobility management»: they have to manage an itinerary (for example a global service chain or a set of similar infrastructure elements), to collect information or to configure parameters on target nodes. After achieving their own process, they have to return the results to the "management node" in charge of the management area (i.e. a particular service chain or a part of the infrastructure). These agents consist in two parts: first an itinerary description and management function and an action part (starting a code agent, installing and starting an action agent...). They are used to collect information on similar infrastructure elements or to get indicator from the different services belonging to a same chain.

- Code agents are in charge of running the indicator building programs to set the complex measures computed from the basic data. These processes can be executed in a distributed way on the different nodes. In this case itinerary agents are used to send computing parameters and bring back the computed results to the area manager.

- Action agents are used to orchestrate the area management: according to the analysis results, these agents are in charge of setting the convenient itinerary, code and action agents.

\section{CONCLUSION}

In this work, in order to enhance dynamic inter-enterprise collaboration based on SOA, we introduced a Virtual Service which correspond to a set of related IT and business services. This Virtual Service is associated to a Service Level Agreements (SLA) and offer also capabilities to manage contracts and to monitor related services. We use a dynamic SLM based on mobile agent orchestration to apply the SLA constraints in order to manage and monitor the execution of services.

Further works will integrate more closely the policy-based service composition process with the enactment of the convenient management system so that management patterns could be identified and re-used to improve the system dynamic enactment.

\section{REFERENCES}

1. Alamri, A., and, M. E. and Saddik, A. E., 2006, Classification of the state-of-the-art dynamic web scrvices composition techniques, International Journal for Wcb and Grid Services, 2(2), pp. 148-166.

2. Ali, L., Mathicu, H. and Biennicr, F., 2006, Monitoring and Managing a Distributed Networks using Mobile Agents, In 2nd IEEE International Conference on Information and Communication Technologics, Damascus, Syria, pp. 3377-3382.

3. Alonso, G., Casati, F., Kuno, H. and Machiraju, V., 2004, Web services Concepts, Architectures and Application, Springer Verlag.

4. Andrews, T., Curbera, F., Dholakia, H., Goland, Y., Klcin, J., Lcymann, F., Liu, K., Roller, D., Smith, D., Thatte, S., Trickovic, I. and Wecrawarana, S., 2003, Business Process Exccution Language for Web Services Version 1.1 available at: http:/www-128.ibm.com/developerworks/ library/specification/ws-bpcl/. 
5. Bartelt, A. and Lamersdorf, W., 2001, A Multi-criteria Taxonomy of Business Models in Electronic Commerce, In Second International WorkshopElectronic Commerce (WELCOM), Heidelberg, Germany, pp. 193-205.

6. Bissel, T., Bogen, M., Bonkowski, C. and Hadamschek, V., 2000, Service level management with agent technology, Computer Networks, 34(6), pp. 831-841.

7. Casati, F. and Shan, M., 2001, Dynamic and Adaptive Composition of e-Services, Information Systems, 6(3).

8. Chaari, S., Biennier, F., Benamar, C. and Favrel, J. (2006) In Knowledge Enterprise: Intelligent Strategies in Product Design, Manufacturing, and Management, Shanghai, China, pp. 920-925.

9. Choi, B., Raghu, T. S. and Vinze, A., 2004, Addressing a standards creation process: a focus on ebXML, International Journal of Human-Computer Studies, 61(5), pp. 627-648.

10. Engel, F., 1999, The role of service level agreements in the internet service provider industry, International Journal of Network Management, 9(5), pp. 299-301.

11. Grefen, P., Aberer, K., Hoffner, Y. and Ludwig, H., 2000, CrossFlow: Cross-Organizational Workflow Management in Dynamic Virtual Enterprises, International Journal of Computer Systems Science \& Engineering, 15(5), pp. 277-290.

12. Kutvonen, L., Metso, J. and Ruokolainen, T., 2005, Inter-enterprise collaboration management in dynamic business networks, In International Conference on Cooperative Information Systems (CoopIS2005), Agia Napa, Cyprus, pp. 593-611.

13. Lazcano, A., Alonso, G., Schuldt, H. and Schuler, C., 2000, The WISE approach to electronic commerce, International Journal of Computer Systems Science \& Engineering, special issue on Flexible Workflow Technology Driving the Networked Economy, 15(5), pp. 345-357.

14. Papazoglou, M., 2003, Service oriented computing: concepts, characteristics and directions, In the 4th IEEE International Conference on Web Information Systems Engineering, Italy, pp. 3-12.

15. Sundaram, M. and Shim, S. S. Y., 2001, Infrastructure for B2B Exchanges with RosettaNet, In Third International Workshop on Advanced Issues of E-Commerce and Web-Based Information Systems, pp. 110-119.

16. TheOpenGROUP, 2004, SLA Management Handbook, available at: http://www.opengroup.org/ pubs/catalog/g045.htm.

17. Verma, D., 1999, Service Level Agreements on IP Networks, Macmillan Technical Publishing.

18. Wob, W., 2000, XML and Meta Data Based EDI for Small Enterprises, In 11 th International Conference on Database and Expert Systems Applications (DEXA 2000), London, UK, pp. 357365 . 